\section{EXPLORING THE CLINICAL ASPECTS OF VACCINE-PREVENTABLE INVASIVE AND NON-INVASIVE BACTERIAL DISEASE WITHIN EUROPE}

\section{A. Vergison}

\section{ULB, Brussels, Belgium}

Bacterial infections remain the most common cause of mortality and morbidity in children worldwide. Implementation of vaccination programmes has decreased the incidence of bacterial disease caused by Haemophilus influenzae type $\mathrm{b}^{1}$ and group $\mathrm{C}$ meningococci; ${ }^{2}$ however, invasive disease and mucosal respiratory tract infections due to Gram-positive bacteria, primarily Streptococcus pneumoniae, remain prevalent. ${ }^{3}$ Pneumonia remains a leading cause of morbidity and mortality in Europe, causing an estimated 300,000 hospitalisations and 31,500 child deaths annually. ${ }^{4}$

S. pneumoniae causes invasive pneumococcal disease (IPD), characterised by bacteraemia, septicaemia and bacterial meningitis. The consequences can be severe, resulting in hospitalisation, complications and death. Whilst non-invasive diseases are generally less severe than IPD, their higher incidence poses a significant healthcare and social burden, including promotion of antibiotic resistance. ${ }^{5}$ The introduction of pneumococcal conjugate vaccines has led to important decreases in the incidence of IPD, ${ }^{6}$ and to a lesser extent on pneumonia hospitalisations ${ }^{7}$ and on acute otitis media (AOM) visits. ${ }^{8}$

Although non-typeable $\mathrm{H}$. influenzae (NTHi) has been increasingly recognised as a pathogen in invasive diseases, ${ }^{9}$ it is also a causative agent of mucosal disease, predominantly in association with $\mathrm{S}$. pneumoniae. The role of NTHi is well established in $\mathrm{AOM},{ }^{10}$ still controversial in lobar pneumonia, and increasingly implicated in chronic bronchial inflammation in young children with persistent wheezing. ${ }^{11}$

In Europe, the spectrum of invasive and non-invasive disease that could be prevented by vaccination remains significant, with potential immediate effects on morbidity and mortality, and long-term impact on indirect disease burden.

\section{References:}

1) Peltola H. Clin Microbiol Rev 2000; 13: 302-17.

2) Trotter CL, et al. FEMS Microbiol Rev 2007; 31:
101-7. 3) Cartwright K. Eur J Pediatr 2002; 161: 188-95. 4) Rudan I, et al. Bull World Health Organ 2008; 86: 408-16. 5) Vergison A, et al. Lancet Infect Dis 2010; 10: 195-203. 6) Pilishvili T, et al. J Infect Dis 2010; 201: 32-41. 7) Grijalva CG, et al. Lancet 2007; 369: 1179-86. 8) Taylor S, et al. $28^{\text {th }}$ Annual ESPID Meeting. Nice, France, 2010: abstract 1334. 9) Slack M, et al. $28^{\text {th }}$ Annual ESPID Meeting. Nice, France, 2010: abstract 153. 10) Vergison A. Vaccine 2008; 26: G5-10. 11) De Schutter I, et al. $27^{\text {th }}$ Annual ESPID Meeting. Brussels, Belgium, 2009: abstract P445.

\section{2}

\section{SYNFLORIX'TM : WHAT HAVE WE LEARNED FROM IMMUNOGENICITY STUDIES?}

\section{J. Wysocki}

\section{Poznan University of Medical Sciences, Poznan, Poland}

Synflorix ${ }^{\mathrm{TM}}$ (10-valent pneumococcal conjugate vaccine; GlaxoSmithKline Biologicals, Belgium) has been investigated globally to evaluate immunogenicity, safety and reactogenicity with different vaccination schedules and routine coadministered vaccines. ${ }^{1}$ As expected, population variability in immune responses following priming was observed between continents. ${ }^{2,3}$ In European studies, Synflorix ${ }^{\mathrm{TM}}$ was proven to be immunogenic post-primary and post-booster vaccination in terms of

-amount of antibody (ELISA) for the 10 serotypes and protein $D$ (carrier for $8 / 10$ serotypes) and

-functional antibodies (opsonophagocytic activity; OPA) for the 10 serotypes. ${ }^{1}$ Furthermore, Synflorix ${ }^{\mathrm{TM}}$ induced similar immunological memory following the licensed $3+1$ schedule or the investigational $2+1$ schedule in Europe, with robust immune responses after a challenge dose 2 years post-booster. ${ }^{4}$

Although not indicated for non-vaccine-serotypes, in clinical trials, Synflorix ${ }^{\mathrm{TM}}$ has induced crossreactive immune responses (ELISA and OPA) and immunological memory against serotypes $6 \mathrm{~A}$ and $19 \mathrm{~A}$ post-primary ${ }^{4}$ and post-booster vaccination. ${ }^{5}$ An 11-valent prototype vaccine included serotype 3; however, no efficacy or immune memory was observed $^{6}$ and it was removed from the final formulation. ${ }^{1}$ Synflorix ${ }^{\mathrm{TM}}$ decreased nasopharyngeal carriage of vaccine-serotypes in the second year of life by $23-35 \%$. $^{7}$ Whilst a trend towards increased carriage of non-vaccine-serotypes was observed, ${ }^{7}$ 
Satellite Symposia Abstracts

a trend towards decreased $6 \mathrm{~A}$ carriage was noted in line with the cross-reactive immune response induced by Synflorix ${ }^{\mathrm{TM}}{ }^{8}$ These data give the first indications of biological activity and effectiveness of Synflorix ${ }^{\mathrm{TM}}$. The efficacy of pneumococcal vaccines needs careful monitoring and ongoing trials (COMPAS $^{9}$ and FinIP ${ }^{10}$ ) will evaluate efficacy and effectiveness, respectively, of Synflorix ${ }^{\mathrm{TM}}$ against invasive pneumococcal disease, pneumonia, acute otitis media and carriage.

\section{References:}

1) Prymula R, et al. Expert Rev Vaccines 2009; 8: 1479-500.

2) Schuerman L, et al. 7th ISPPD meeting. Tel Aviv, Israel, 2010: abstract 523.

3) Schuerman L, et al. 7th ISPPD meeting. Tel Aviv, Israel, 2010: abstract 517.

4) Schuerman L, et al. 7th ISPPD meeting. Tel Aviv, Israel, 2010: abstract 475.

5) Silfverdal SA, et al. 7th ISPPD meeting. Tel Aviv, Israel, 2010: abstract 633.

6) Prymula R, et al. Lancet 2006; 367: 740-8.

7) Prymula R, et al. 6th WSPID meeting, Buenos Aires, Argentina, 2009: abstract 1113.

8) Prymula R, et al. $28^{\text {th }}$ Annual ESPID Meeting. Nice, France, 2010: abstract 259.

9) COMPAS (http://clinicaltrials.gov identifier: NCT00466947).

10) FinIP (http://clinicaltrials.gov identifier: NCT00861380).

\section{3}

\section{PREVENTING ACUTE OTITIS MEDIA THROUGH VACCINATION: WHAT MIGHT THE FUTURE HOLD}

\section{Knuf}

\section{Universal Medicine, Mainz, Mainz, Germany}

Acute otitis media (AOM) constitutes a significant burden in children, in terms of clinical, psychological, social and economic costs. ${ }^{1,2}$ It is the most frequent reason for antibiotic prescriptions in children, ${ }^{3}$ thus contributing to the development of antibiotic resistance. Therefore, preventing AOM via vaccination is a promising approach. ${ }^{4}$ The impact of vaccination with the 7-valent pneumococcal conjugate vaccine (PCV7) on AOM visits and episodes in the US has been reported in various studies. There is a broad range of reported impacts, with randomised controlled trials reporting 6-9\% decreases and most observational studies reporting $13-26 \%$ decreases; ${ }^{5}$ however, one study reported a substantially higher decrease (43\%). ${ }^{6}$ The $43 \%$ reduction was well outside the range reported in the otherstudies, possibly due to various methodological limitations.

Synflorix ${ }^{\mathrm{TM}}$ (10-valent pneumococcal conjugate vaccine; GlaxoSmithKline Biologicals, Belgium) is licensed in Europe for active immunisation against invasive disease and AOM caused by Streptococcus pneumoniae in infants and children from 6 weeks up to 2 years of age. ${ }^{7}$ Although not indicated for protection against AOM caused by non-typeable Haemophilus influenzae (NTHi) in Europe, there are data that support the hypothesis that this vaccine may also protect against AOM due to NTHi; these include data from the POET trial indicating efficacy of $33.6 \%, 51.5 \%$ and $35.3 \%$ for all-cause AOM, pneumococcal AOM and NTHi AOM, respectively; ${ }^{8}$ immunogenic bridging studies of Synflorix ${ }^{\text {TM }}$ to the POET trial; ${ }^{9}$ and pre-clinical efficacy data in chinchillas. ${ }^{10}$ Importantly, clinical efficacy (COMPAS) and effectiveness (FinIP) trials are ongoing to evaluate potential indications for AOM and pneumonia.

\section{References:}

1) Melegaro A, et al. J infect 2006; 52: 37-48.

2) Daly KA, et al. Pediatr Rev 1999; 20: 85-93.

3) Schindler C, et al. Pharmacoepidemiol Drug Saf 2003; 12: 113-20.

4) Vergison A, et al. Lancet Infect Dis 2010; 10: 195-203.

5) Taylor S, et al. $28^{\text {th }}$ Annual ESPID Meeting. Nice, France, 2010: abstract 1334.

6) Zhou F, et al. Pediatrics 2008; 121: 253-60.

7) Synflorix ${ }^{\mathrm{TM}}$ Summary of Product Characteristics. Available from: http://www.emea.europa. eu/humandocs/PDFs/EPAR/synflorix/emeacombined-h973en.pdf [Accessed May 2010].

8) Prymula R, et al. Lancet 2006; 367: 740-8. 